\title{
Harmonically Trapped Classical Gas under Critical Rotation
}

\author{
J. ChWedeńczuK ${ }^{a}$, P. Zińa $a$, TrippenbaCh ${ }^{a}$, \\ B. DAZBROWSKA ${ }^{b}$, M. GAJDA ${ }^{c}$ AND K. RzĄŻEWSKI ${ }^{b, d}$ \\ ${ }^{a}$ Institute of Experimental Physics, Warsaw University \\ Hoża 69, 00-681 Warsaw, Poland \\ ${ }^{b}$ Cardinal Wyszyński University, al. Lotników 32/46, 02-668 Warszawa, Poland \\ ${ }^{c}$ Institute of Physics, Polish Academy of Sciences \\ al. Lotników 32/46, 02-668 Warszawa, Poland \\ ${ }^{d}$ Center for Theoretical Physics, Polish Academy of Sciences \\ al. Lotników 32/46, 02-668 Warszawa, Poland \\ (Received April 28, 2003; revised version August 11, 2009)

\begin{abstract}
We study one- and two-dimensional systems of two interacting particles in a time dependent harmonic potential. In a case of one-dimensional geometry a frequency of the potential varies periodically, while in the two-dimensional case the harmonic potential rotates with a constant angular velocity. We show that depending on the driving frequency the distance between the particles can either explode or stay bound. Repulsive interaction can prevent the explosion, which seems quite counter-intuitive. Our work is related to Ecole Normale Supérieure experiment and shows that the effect found there is purely classical.
\end{abstract}

PACS numbers: 03.75.-b, 03.75.Fi

\section{Introduction}

Seven years after the achievement of Bose-Einstein condensation in trapped ultra-cold atomic gases, new impressive experiments are reported. They stimulate a progress in the array of dynamics of quantum degenerate gases. In this paper we study classical and quantum models of interacting particles subject to the parametric resonance [1-6]. Our study was triggered by the recent experiment of the Ecole Normale Supérieure group [7], in which a gas of ultra-cold atoms trapped in a harmonic potential was exposed to a critical rotation, i.e. rotation with a frequency close to the trapping frequency. In the experiment two different types of dynamics had been identified: 
- Explosion, where under the influence of the rotations the cloud expands in one direction. This happens for a dilute cloud at a temperature above a critical one.

- The regime, where the cloud spirals out of the trap as a rigid body. This situation takes place for the Bose-Einstein condensate with the sufficiently strong repulsive interaction.

This behavior is typical of the parametric resonance, but it refers now to the internal degrees of freedom of the system rather than to the motion of the center of mass. Clearly atom-atom interactions modify the dynamics, but the effect is counter-intuitive: repulsive interactions (important when the kinetic energy becomes small) prevent spreading of the condensate. Some insight may be obtained from approximate analytic solution of the Gross-Pitaevskii (GP) equation. Authors of paper [7], using an Ansatz wave function

$$
\psi(x, y) \propto \exp \left(\mathrm{i} \alpha x y-\beta x^{2} / 2-\gamma y^{2} / 2\right)
$$

and extremizing the GP energy functional with respect to the real parameters $\alpha, \beta$, and $\gamma$ explained the behavior of the gas in the experiment and attributed a change of the stability of the system to the frequency shift of the quadruple mode. This shift drives the system out of the resonance with the rotation frequency.

In this paper we study the simple classical and quantum models of interacting particles and we show that the effect of stabilization of the atomic condensate expansion is a purely classical phenomenon. The paper is organized as follows:

In Sect. 1 we study a classical one-dimensional system of two interacting particles, confined in the external harmonic potential with a modulated frequency. We show that the stability conditions of such dynamics depend crucially on the inter-atomic interaction. To prove this point we compare the results of the classical dynamics in two cases: (a) non-interacting atoms; (b) atoms repelling each other via a Gaussian potential.

In Sect. 2 we analyze a two-dimensional system of two particles in the rotating harmonic trap. We solve both the Schrödinger equation and the Newton equation for the relative coordinate in the rotating frame of reference. We show that both approaches lead to qualitatively similar results. This model directly refers to the experiment [7] and resembles the results based on the GP equation.

\section{One-dimensional model}

We start with a one-dimensional model. Let us consider a system of two particles of mass $m$ interacting by the repulsive force $f(x)$ and confined by the external harmonic potential of frequency $\omega$. Additionally, this frequency is modulated periodically and we denote by $\epsilon$ and $\gamma$ an amplitude and a frequency of modulation, respectively. The Newton equations of motion read

$$
\ddot{x}_{1}+[1+\epsilon \cos (\gamma t)] x_{1}=f\left(x_{1}-x_{2}\right),
$$




$$
\ddot{x}_{2}+[1+\epsilon \cos (\gamma t)] x_{2}=-f\left(x_{1}-x_{2}\right),
$$

where $x_{1}$ and $x_{2}$ are the coordinates of the first and second particle, respectively, and $\omega$ defines the unit of frequency, time is measured in units of $\tau=1 / \omega$. For simplicity we consider a Gaussian inter-particle potential, hence the expression for the mutual repulsive force $f(x)$ takes the form

$$
f(x)=\frac{1}{2} g x \exp \left(-\frac{1}{2} x^{2}\right),
$$

where $g$ is the interaction strength. Upon separating the center of mass motion (which is not affected by the inter-atomic interaction) and relative motion, we obtain for the relative coordinate $x_{\mathrm{r}}=x_{1}-x_{2}$ the following equation:

$$
\ddot{x}_{\mathrm{r}}+[1+\epsilon \cos (\gamma t)] x_{\mathrm{r}}=g x_{\mathrm{r}} \exp \left(-\frac{1}{2} x_{\mathrm{r}}^{2}\right) .
$$

We now present the results of the numerical analysis of (4). The solution $x_{\mathrm{r}}(t)$ depends on three parameters: $\epsilon, \gamma$, and $g$. According to long time behavior we classify the solutions into two classes

- exploding - when the distance between the particles is not bounded,

- bound - when the distance between the particles does not exceed a certain value.

The results of numerical analysis of the one-dimensional case are summarized in Figs. 1a and b, where we vary $\gamma$ and $\epsilon$ with $g$ fixed. We mark the unstable solutions in black. We have chosen the initial conditions $x_{\mathrm{r}}(0)=1$ and $\dot{x}_{\mathrm{r}}(0)=0$, and the criterion, according to which the solution is considered to be exploding (unstable), if during $500 \tau$ the absolute value $x_{\mathrm{r}}(t)$ exceeds 10 . Comparing Figs. 1a (with $g=0$ ) and b (with $g=5$ ) we conclude that when we turn on the interactions, the region of unstable solutions in the $\gamma-\epsilon$ plane is shifted towards higher values of $\epsilon$ and towards smaller values of $\gamma$. Both effects have a clear meaning. The shift towards smaller values of $\gamma$ is caused by the change of resonance frequency of the system when we turn on the interaction between particles.
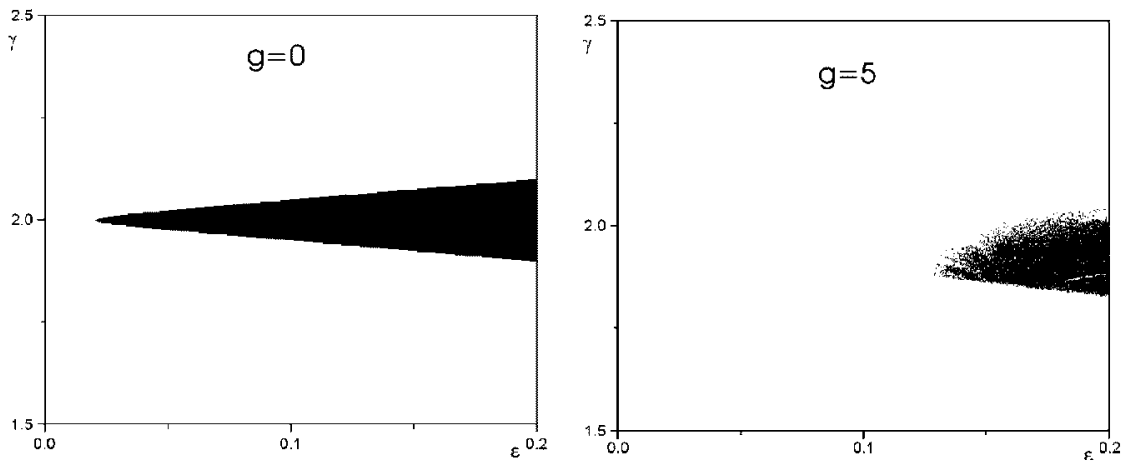

Fig. 1. Stability diagram for the non-interacting $(g=0)$ (a) and weakly interacting $(g=5)$ (b) systems in the $\epsilon-\gamma$ variables. Instability regions are marked in black. 
The phenomenon which we call an explosion of the trajectories will occur for $g \neq 0$ at different frequency, a doubled new resonance frequency of the system. It follows from the general theory of the parametric resonance that we should observe the explosion at the frequencies $2 \gamma / n$, where $n$ is an arbitrary integer, but the one at $2 \gamma$ is the most pronounced. The shift towards higher values of $\epsilon$ is a manifestation of the increase in stability of the system with the increase in the interaction strength and is also present in the work of Rosenbuch et al. [7]. We would like to stress that the shape of the black region depends on the choice of initial conditions, but the general trends pointed out above are general.

\section{Two-dimensional model}

Now we turn to the simulations of the dynamics of a two-dimensional system. Two-dimensional geometry allows us to incorporate a rotation of the trap and therefore to mimic the condition of the experiment [7]. We will, however, still consider a two-particle system, or more precisely two interacting particles in the rotating harmonic trap. This restriction allows for explicit treatment of two-body interactions without the mean field approximation which leads to the Gross-Pitaevskii equation. We assume that the particles repel each other by model forces described by a Gaussian-like potential

$$
V_{\text {int }}=g \exp \left(-\frac{r^{2}}{2 l_{0}^{2}}\right)
$$

where $g$ is a parameter describing the interaction strength, while $r=(x, y)$ is a relative coordinate. The parameter $l_{0}$ determines an effective range of the potential. We set its value to $l_{0}=0.4 a_{\mathrm{HO}}$, where $a_{\mathrm{HO}}=\sqrt{\hbar /(m \omega)}$. In the following we use oscillatory units, i.e. the unit of distance is $a_{\mathrm{HO}}$, the unit of time is $\tau=1 / \omega$, and the unit of energy is $\hbar \omega$.

Since the center of mass and relative coordinate separate and interaction potential does not influence the center of mass motion we will only consider the dynamics of the relative coordinate. It is governed by the following Hamiltonian:

$$
\begin{aligned}
H= & \frac{1}{2}\left(p^{2}+r^{2}\right)+g \exp \left(-\frac{r^{2}}{2 l_{0}^{2}}\right) \\
& +\frac{1}{2} \epsilon\left[\left(y^{2}-x^{2}\right) \cos (2 \Omega t)-2 x y \sin (2 \Omega t)\right],
\end{aligned}
$$

where $\Omega$ is the rotation frequency and $\epsilon$ is a parameter that measures an anisotropy of the rotating potential. Notice that if we want to establish a correspondence between our one- and two-dimensional models we have to put $\gamma=2 \Omega$. In our simulations we assume that an initial state is a ground state of the time independent part of the Hamiltonian (6), i.e. we assume that initially the rotation is turned off and $\epsilon=0$. We determine the initial wave function and its energy $\mu$ (for any particular value of $g$ ) by numerically solving the corresponding stationary Schrödinger equation. Thus the generated initial state is then evolved with 
the full time dependent Hamiltonian (6), transformed to the frame rotating with the frequency $\Omega$. Hence we assume that the rotation is turned on the very short time scale, short in comparison with the characteristic time of the wave function dynamics.

Parallel to this quantum mechanical version of the simulations we perform a corresponding classical one. In the latter we solve the classical equations of motion derived from the classical Hamiltonian of the form (6). In this case we specify the initial conditions by choosing $N=1000$ initial phase-space points selected according to the classical microcanonical distribution, i.e. with the phase-space density proportional to

$$
\rho \sim \delta(H(p, r)-\mu)
$$

with an additional condition of zero angular momentum, $\boldsymbol{L}=r \times p=0$. The phase-space distribution obtained this way is analogous to the quantum probability density $\left(\left|\psi_{0}(r)\right|^{2}\right)$. We compare results obtained in the quantum and classical calculations. The similar phase-space averaging methods have been used for description of an ionization process of the hydrogen atom [8].

In Fig. 2 classical and quantum spatial distributions at a time $t=20 \tau$ are shown in the case of weak interactions $(g \ll 1)$. The final distribution becomes elongated in one direction. As we see, under these conditions both approaches predict explosion of the system under the parametric resonance rotation. It can be easily understood, if we keep in mind, that in the absence of repulsive interaction the system is unstable in the following range of rotation frequencies:

$$
\sqrt{1-\epsilon}<\Omega<\sqrt{1+\epsilon} .
$$

If the initial kinetic energy of the system is much larger than the interaction energy then the above condition (8) defines the region of unbound (unstable) motion. This is a reason for the explosion observed in Fig. 2. We expect, however, that when the strength of repulsive interaction increases and the rotation frequency remains constant, the system will eventually become stable, according to the indications that we obtained from the one-dimensional model. It happens at the expense of the change of the resonance frequency of the system, which depends on the interaction strength. As we see in Fig. 3, which shows the dynamics for much greater value of $g$ the quantum and classical distributions again generate quite similar results. The two particles cannot be found close to one another because of the repulsive interactions. This is the origin of a central hole visible in the figure. If we look at the single-particle density, which is proportional to the probability of finding one particle at a given space point, regardless the position of the second one, we would not observe such a feature.

In order to systematically check the stability of the system within the classical approach we define the "explosion" probability $P_{\mathrm{e}}$ as a fraction of the "exploding" trajectories $N_{e} ; P_{\mathrm{e}}=N_{\mathrm{e}} / N$. The trajectory is considered to be "exploding" if after some time $T$ the final amplitude of relative coordinate exceeds a certain 

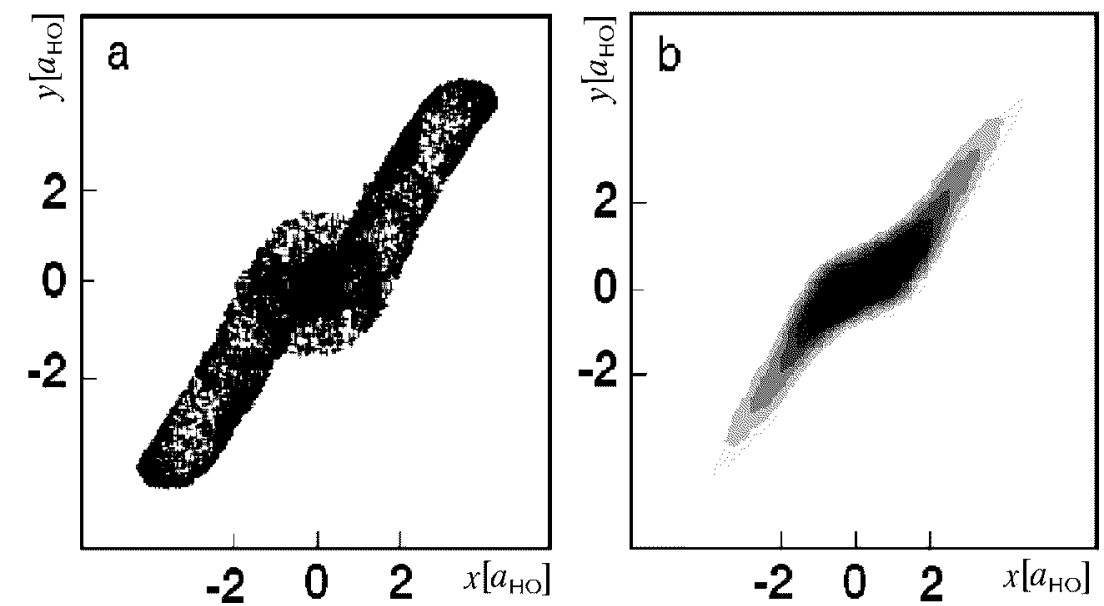

Fig. 2. Classical (a) and quantum (b) spatial density in the relative coordinate space for $g=1, \epsilon=0.2, \Omega=1$ at the time $t=20 \tau$.
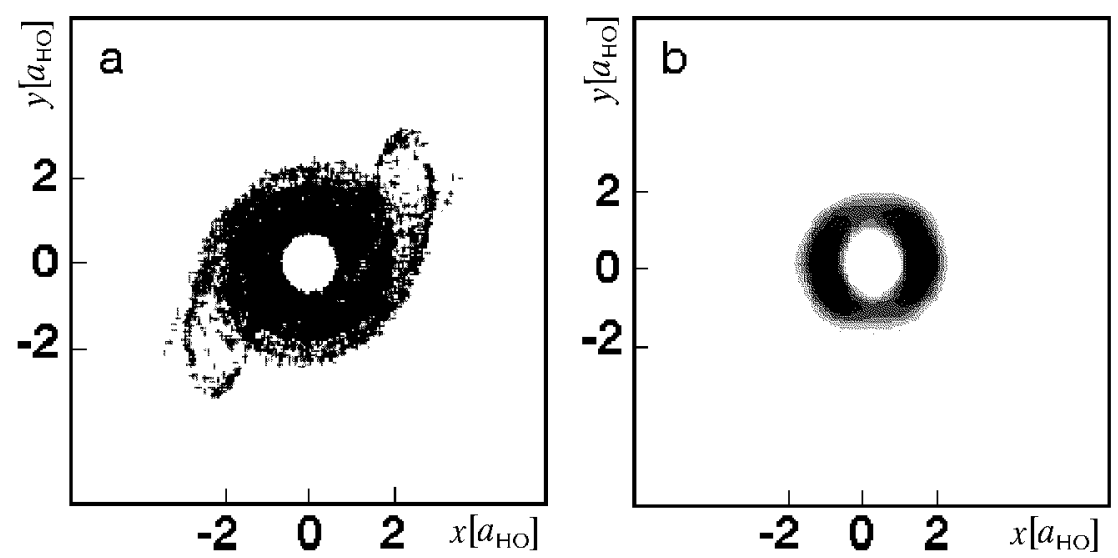

Fig. 3. Classical (a) and quantum (b) spatial density in the relative coordinate space for $g=50, \epsilon=0.2, \Omega=1$ at the time $t=20 \tau$.

value $r(T)>R$. We arbitrarily set values of these parameters to $T=100 \tau$ and $R=5$. Our results very weakly depend on the choice of these parameters.

In Fig. 4 we present the explosion probability in the space of parameters $\Omega$ and $g$ for two values of the parameter $\varepsilon=0.1$ and $\varepsilon=0.2$. In the first case the unstable region (the black area) diminishes with increasing interaction strength. If the interaction strength exceeds the critical value of $g \simeq 3.0$ all trajectories are bound in the whole range of rotation frequencies.

The situation is different for larger anisotropy $\varepsilon=0.2$. The unstable region shrinks with increasing $g$ but never disappears. In addition, in a relatively wide region of frequencies the escape probability is larger than zero but significantly smaller than 1. We can distinguish therefore, two different kinds of instability. 

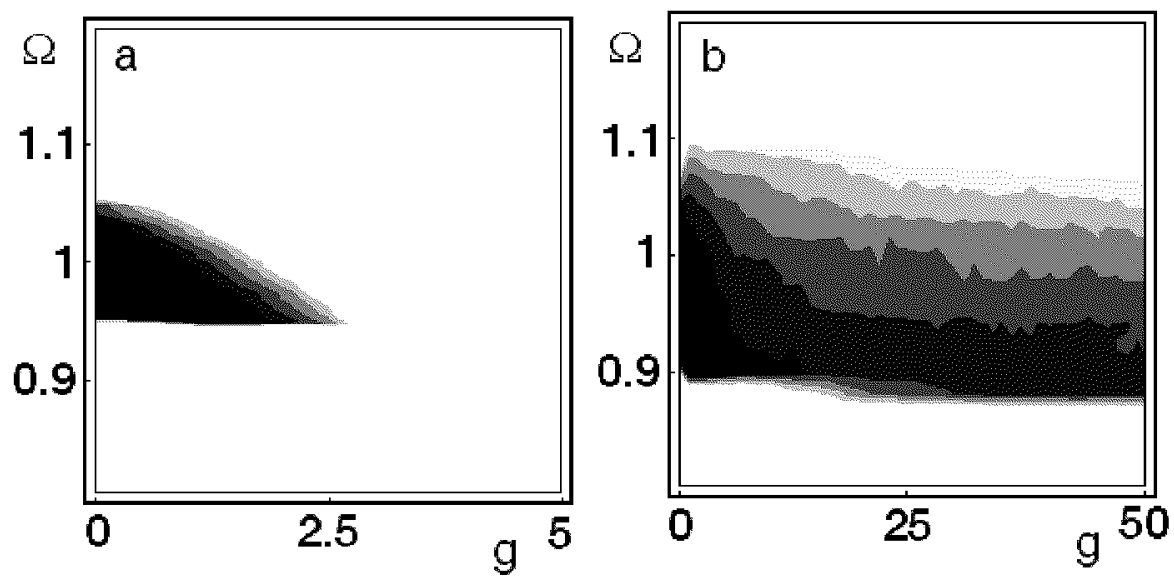

Fig. 4. Stability diagram: (a) $\varepsilon=0.1$; (b) $\varepsilon=0.2$. The darkest area corresponds to a fully unstable regime while areas of a different gray scale intensity correspond to a partial instability.
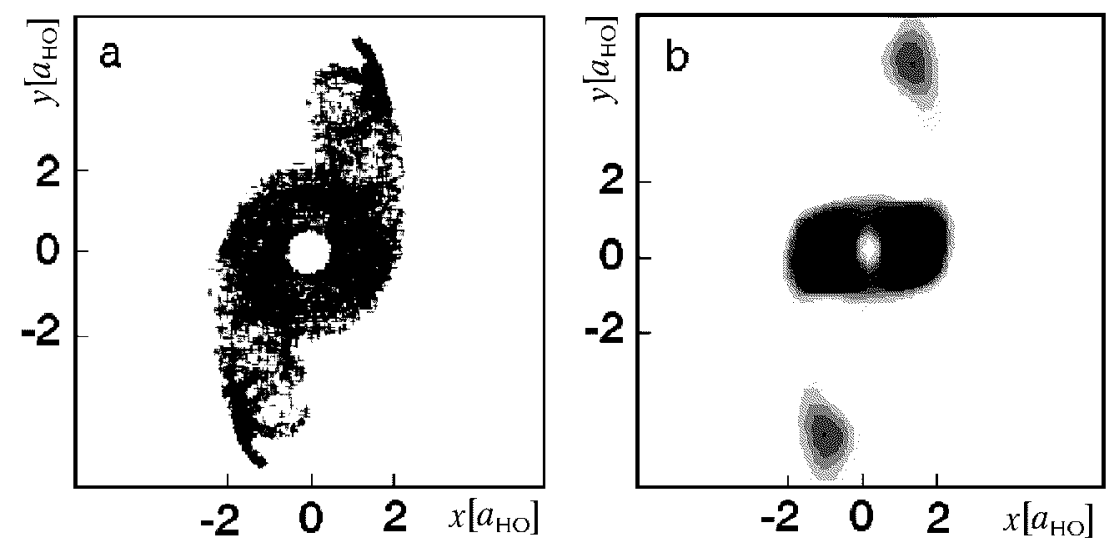

Fig. 5. Classical (a) and quantum (b) spatial density in the relative coordinate space for $g=10, \epsilon=0.2, \Omega=0.88$. Note that some fragments separate and leave the area of the central cloud.

The first one - almost all considered trajectories are unbound, $P_{\mathrm{e}} \simeq 1$. This is the darkest region in Fig. 4. The second is the one, where only a fraction of initial data corresponds to unbound motion. This situation corresponds to different intensities of the gray scale in the figure. In particular the light gray colour corresponds to $P_{\mathrm{e}}<0.1$. A typical final density distribution in such a situation is shown in Fig. 5. Many trajectories stay bound while a few trajectories are exploding. The system breaks into fragments. This scenario is also valid in the quantum treatment. However, such solutions cannot be described by the Ansatz assumed 
in [7]. To compute the single particle probability density we have to integrate over all positions of its companion:

$$
\left|\Psi\left(\boldsymbol{r}_{1}, t\right)\right|^{2}=\int \mathrm{d} \boldsymbol{r}_{2}\left|\Psi_{\mathrm{rel}}^{*}\left(\boldsymbol{r}_{1}-\boldsymbol{r}_{2}, t\right) \Psi_{\mathrm{CM}}\left(\boldsymbol{r}_{1}+r_{2}, t\right)\right|^{2},
$$

where $\Psi_{\text {rel }}\left(\boldsymbol{r}_{1}-\boldsymbol{r}_{2}, t\right)$ is a wave function of relative coordinate. It is a solution of the time-dependent Schrödinger equation with the Hamiltonian given by Eq. (6) and with a substitution $\boldsymbol{r}:=\boldsymbol{r}_{1}-\boldsymbol{r}_{2} . \Psi_{\mathrm{CM}}\left(\boldsymbol{r}_{1}+\boldsymbol{r}_{2}, t\right)$ is a wave function of the center of mass which is the ground state of the trapping potential. Figure 6 shows the single particle density for the same set of parameters as in Fig. 5. It is clear that the single particle density distribution is in some respect similar to the relative coordinate density distribution. In both cases one observes that pieces of the wave function are detached from the central part, while the shape of the central part of wave function does not substantially differ from those obtained in the stable region. This situation resembles the behavior of the system in the classical limit, where only fraction of the trajectories in the phase space are stable.

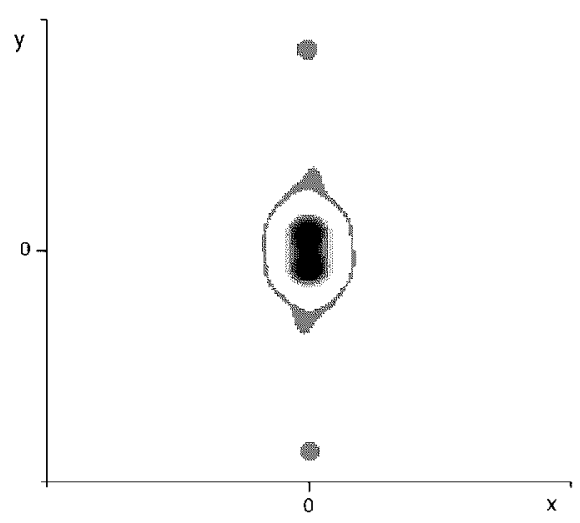

Fig. 6. Density of single-particle wave function for the parameters as in Fig. 5.

This almost classical behavior of the quantum wave function does depend on the interatomic interaction potential. We tested the solutions of the Gross-Pitaevskii equation both in the regime of the parameters analogous to that used above and outside this region. In all the cases where we performed the test, the dynamic of the wave function agreed well with the self-similar solutions and no parts of the wave function were detached.

In conclusions, we performed classical and quantum stability analyses of the two-particle system close to the parametric resonance. The analysis presented here proved that the stabilization of the expanding wave function in the rotating harmonic trap due to the interatomic interactions observed in the experiment of Rosenbuch et al. [7] has a purely classical analog. In addition we have identified regimes of parameters when some small fragments of the initial cloud escape in a 
certain direction while most of the system survives the explosion. This property was not found in the Bose-Einstein condensate dynamics.

\section{Acknowledgment}

The authors would like to acknowledge support from the State Committee for Scientific Research Grant 2P03 B7819 and (M. T.) PBZ/KBN/043/P03/2001. The work is a part of a general program on cold-atom physics of the National Laboratory of AMO Physics in Torun, Poland.

\section{References}

[1] N.M. McLachlan, Theory and application of Mathieu functions, Clarendon Press, Oxford 1947.

[2] N.N. Bogoliubov, Y.A. Mitropolsky, Asymptotic Methods in the Theory of Non-linear Oscillations, Gordon and Breach Science Publishers, New York 1961.

[3] C. Hayashi, Nonlinear Oscillations in Physical Systems, Princeton University Press, Princeton 1985.

[4] V.V. Bolotin, The Dynamic Stability of Elastic Systems, Holden-Day, San Francisco 1964.

[5] L. Salasnich, Laser Physics 13, 547 (2003).

[6] Yu. Kagan, L.A. Maksimov, e-print cond-mat/0212377.

[7] P. Rosenbuch, D.S. Petrov, S. Sinha, F. Chevy, V. Bretin, G. Schlyapnikow, J. Dalibard, Phys. Rev. Lett. 88, 250403 (2002).

[8] J.G. Leopold, I.C. Percival, Phys. Rev. Lett. 41, 944 (1978). 